\title{
Anomalies of hypersound velocity and attenuation in ferroelectric tris-sarcosine calcium chloride (TSCC) for small-angle and right-angle Brillouin scattering and Brillouin backscattering
}

\author{
Jin Tong Wang and V. Hugo Schmidt \\ Department of Physics, Montana State University, Bozeman, Montana 59717
}

(Received 13 December 1985)

\begin{abstract}
The Brillouin spectra of ferroelectric tris-sarcosine calcium chloride have been observed using small-angle and right-angle scattering and also backscattering. For different-frequency phonons along the same direction, analogous anomalies in the sound velocity and the attenuation are seen. The smallest angle we have achieved is $7.48^{\circ}$. The temperature and frequency dependences of the sound velocity are discussed. The fact that the linewidth maximum for [001] phonons occurs somewhat below $T_{c}$ seems to indicate that the anomalies are due to piezoelectric coupling induced by spontaneous polarization below $T_{c}$. For [010] phonons the elementary relaxation times which relate to the energy are estimated as $\tau_{0}^{E}=5.25 \times 10^{-13} \mathrm{sec}$ above $T_{c}$ and $\tau_{0}^{E}=3.32 \times 10^{-12} \mathrm{sec}$ below $T_{c}$. The phonon attenuations are also estimated and compared with the observed ones. For the [001] phonons the elementary relaxation time is estimated as $\tau_{0}=5.25 \times 10^{-14} \mathrm{sec}$, in good agreement with the value obtained from right-angle Brillouin scattering.
\end{abstract}

\section{INTRODUCTION}

Tris-sarcosine calcium chloride (TSCC), formula $\left(\mathrm{CH}_{3} \mathrm{NHCH}_{2} \mathrm{COOH}\right)_{3} \mathrm{CaCl}_{2}$, was found to exhibit a ferroelectric (FE) phase transition at the Curie point, 130 K. ${ }^{1(a)}$ At that time it was claimed that the Curie point for this crystal was $127 \mathrm{~K} .^{1(\mathrm{~b})}$ The crystal structure of TSCC is significant for both dielectric and the ultrasonic velocity anomalies. The TSCC crystal is orthorhombic with lattice constants $a=9.156 \pm 0.01, b=17.460 \pm 0.045$, and $c=10.265 \pm 0.005 \AA$, and with $Z=4$ formula units in the unit cell. The space group is Pnma $\left(D_{2 h}^{16}\right)$ in the paraelectric (PE) phase and $P_{n 2} a\left(C_{2 h}^{9}\right)$ in the FE phase. ${ }^{2}$ According to the structural analysis by Ashida et al., the crystal structure of TSCC is pseudohexagonal when viewed down the a axis. ${ }^{3}$ There are 12 sarcosine zwitterion molecules in the unit cell. They are of two types. One comprises the four molecules in the mirror plane. The other eight molecules in general positions (type 2) are slightly bent. There are three kinds of $\mathrm{N}-\mathrm{H} \cdots \mathrm{Cl}$ hydrogen bonds present in TSCC, one with a total bond length of $3.18 \AA$ and two with $3.22 \AA$, but with different $\mathrm{N}-\mathrm{H}$ distances. ${ }^{4}$

Since the crystal at room temperature belongs to the orthorhombic system, it is optically biaxial. The acute bisectrix is the a axis, the optical plane is perpendicular to the $c$ axis, and the crystal is optically positive. ${ }^{5}$

When the crystal undergoes the PE-FE transition, the reflection planes perpendicular to the $\mathrm{FE} b$ axis are suppressed. $^{6}$ The sarcosine molecules in the crystal have the form<smiles>CNCC(=O)O</smiles>

which may have permanent dipole moments because of the $\mathrm{NH}_{2}{ }^{+}$and $\mathrm{COO}^{-}$groups. $^{5}$

The FE transition is a structural phase transition. Such transitions are generally divided into two categories, displacive and order-disorder. Particularly with TSCC there have been strong arguments about which category the FE transition falls into. However, a well-defined limit between these categories does not exist, and we consider that TSCC shows features of both transition types.

Chen and Schaack $^{4}$ are the latest proponents of an order-disorder transition for TSCC. They interpret their infrared and Raman results in terms of a softening of an optical phonon caused by its interaction with an unspecified entity which relaxes rapidly due to thermally activated hopping across a double-minimum well barrier. The protons in hydrogen bonds presumably are ordered in the $\mathrm{N}-\mathrm{H} \cdots \mathrm{Cl}$ configuration at all temperatures because the anions are dissimilar. However, there may be ordering of the mirror-plane sarcosine molecules associated with motion of their methyl or carboxyl groups. ${ }^{5}$ The observation by Sandvold and Courtens ${ }^{6}$ of logarithmic behavior of dielectric susceptibility and spontaneous polarization over a temperature range as wide as $50 \mathrm{~K}$ indicates that there are dipole-dipole interactions in TSCC, in agreement with a report on the specific heat. ${ }^{7}$ It might thus turn out that TSCC is the best current example of uniaxial dipolar behavior in a ferroelectric. The spontaneous polarization is very small whereas $T_{c}$ is large, so the ratio of dipolar energy to thermal energy (calculated by Lajzerowicz and Legrand $^{8}$ for some other ferroelectrics) is quite small, of order 0.01 . Another interesting property of this crystal is that the TO and LO phonons are underdamped and soften at the same temperature, ${ }^{9}$ which was interpreted as indicating that the transition is entirely displacive. These results also indicate that the short-range force and Coulomb force participate in the phase transition almost equally. ${ }^{10}$ 
Since there are such different indications of the strength of the dipole-dipole interaction, studies which may confirm the role of interactions are of particular interest.

One can expect two large anomalies in a Brillouin scattering study of TSCC. First, we can expect large anomalies in the velocity and attenuation of longitudinal phonons propagating along directions perpendicular to the polar axis. ${ }^{11}$ These anomalies are caused by piezoelectric coupling in the FE phase. Because TSCC in the PE phase is centrosymmetric and hence not piezoelectric, this coupling is not intrinsic but rather is induced by the spontaneous polarization. From these anomalies we can evaluate the relaxation time of the polarization of the electric dipole system. ${ }^{12}$ Second, Sorge and Straube ${ }^{11}$ observed that at $20 \mathrm{MHz}$ the longitudinal ultrasonic wave propagating along the polar axis $b$ also shows large anomalies of attenuation and velocity near $T_{c}$. Because of the depolarization effect, in a system with electron-dipole-dipole interactions such as the dipole-dipole interaction along the b axis in TSCC, these anomalies are expected to occur not from piezoelectric coupling but from electrostrictive coupling.

We have performed small-angle and right-angle Brillouin scattering and Brillouin backscattering and have measured the dielectric constant simultaneously when performing the right-angle scattering. The frequency range in which we were working is about $2 \mathrm{GHz}$ (for smallangle scattering) to $30 \mathrm{GHz}$ (backscattering). The smallest angle we used is $7.48^{\circ}$. As far as we know, it is the smallest Brillouin scattering angle ever achieved. As usual, we evaluate the elementary relaxation times for phonons propagating along and perpendicular to the polar axis by investigating anomalies in their velocity and attenuation from Brillouin scattering. A remarkable feature of our experiment is the large frequency range $(2-30$ $\mathrm{GHz}$ ) in which the sound velocity is apparently larger in backscattering than in small-angle and right-angle scattering.

\section{EXPERIMENTAL PROCEDURE}

Single crystals of TSCC were grown from an aqueous solution of sarcosine and calcium chloride by slow cooling. The solution was prepared from chemicals of special quality and was filtered by a $0.2-\mu \mathrm{m}$-pore-size membrane filter. The sample is a carefully polished rectangular parallelepiped. Aluminum was evaporated onto two surfaces of the right-angle-scattering sample as electrodes for measurement of the dielectric constant $\epsilon_{0}$. This sample was annealed at $140^{\circ} \mathrm{C}$ for $20 \mathrm{~h}$ in an evacuated glass tube to improve the quality of the crystal.

The sample for the small-angle scattering and backscattering is also a parallelepiped which is $8 \mathrm{~mm}$ long along the a axis. This sample was not heat-treated.

Each sample was placed in an optical cell described previously. ${ }^{13}$ The optical cell is surrounded by two copper radiation shields with the outer shield connected directly to the liquid-nitrogen reservoir. The temperature in the inner shield was controlled at about $10 \mathrm{~K}$ lower than that of the optical cell. In this way, the temperature in the optical cell could be controlled within $3 \mathrm{mK}$ during the mea- surement using a Lake Shore Cryotronics model CSC-400 temperature controller.

A Lexel model 95-2 argon-ion laser operating in a single mode at a wavelength of $5145 \AA$ and a power level of $300 \mathrm{~mW}$ was used as a light source. In order to keep the difference of temperature between the sample and the temperature sensor constant, we kept the power level of the laser constant. The scattered light was collected in a cone of $0.4^{\circ}$ and analyzed by a piezoelectrically scanned Burleigh model 140 Fabry-Perot interferometer. Finesse optimization and drift control were achieved by a homemade control system using an AIM-65 microcomputer and interface. The period of a single scan of the Fabry-Perot was about $10 \mathrm{~s}$. The signal was stored in the microcomputer and displayed in a multichannel analyzer. The finesse of the spectrometer was typically 42-46. The laser line broadening due to the jittering was claimed by the manufacturer to be about $10 \mathrm{MHz}$ FWHM (full width at half maximum). The angle of the incident light cone was about $0.458^{\circ}$.

For reducing the natural-phonon linewidth, the natural-phonon spectrum and the instrumental function were assumed to have Lorentzian distributions, and the broadening due to collection optics was assumed to have rectangular distribution. In this case, the natural-phonon linewidth $\left(W_{\mathrm{ph}}\right)$ is given by ${ }^{12}$

$$
W_{\mathrm{ph}}=\left(W_{\text {obs }}^{2}-W_{\text {ang }}^{2}\right)^{1 / 2}-W_{\text {inst }},
$$

where $W_{\text {obs }}, W_{\text {inst }}$, and $W_{\text {ang }}$ represent the observed, instrumental, and collection optics linewidths, respectively. In our experiments, $W_{\text {inst }}=0.022 R_{\mathrm{FS}}$ where $R_{\mathrm{FS}}$ is the free spectral range which varied among the different scattering angles.

A bridge composed of an inductor and capacitor was used to measure the dielectric constant. The $1-\mathrm{kHz}$ electrical field was generated by a Princeton Applied Research model 124A lock-in amplifier.

\section{RESULTS AND DISCUSSION}

\section{A. Presentation of results}

Figures 1(a), 1(b), and 1(c) show the temperature dependences of the Brillouin shifts for the longitudinal phonons propagating along [010] at scattering angles $7.48^{\circ}, 90^{\circ}$, and $170^{\circ}$, respectively. Each component shows the anomaly around $T_{c}$ and the temperature dependences are similar. The phonons at small angle and right angle barely show temperature dependence in the PE phase well above $T_{c}$. However, for backscattering, the frequency shift for this phonon shows appreciable temperature dependence both in the high- and low-temperature regions. Our backscattering result is similar to that of Smolensky et al. ${ }^{14}$

Figures 2(a), 2(b), and 2(c) show the analogous temperature dependences of the Brillouin shift for the [001] longitudinal phonons at the three scattering angles. All of them show very strong temperature dependences in both the high- and low-temperature regions. The right-anglescattering results are from Hikita et al. ${ }^{12}$ and the backscattering results are from Smolensky et al. ${ }^{14}$

More details near $T_{c}$ are shown in Figs. 3(a), 3(b), and 

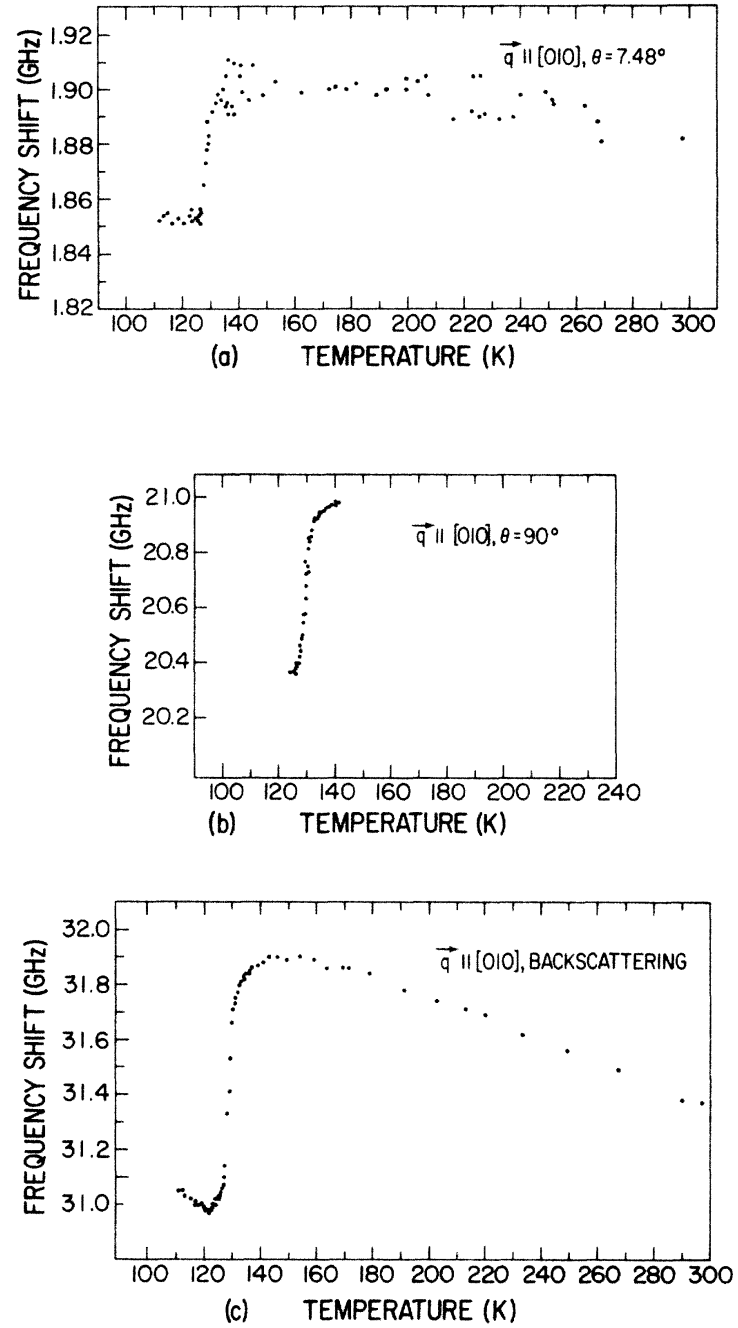

FIG. 1. Temperature dependences of Brillouin shifts of longitudinal phonons propagating along the $b$ axis for (a) small-angle scattering, (b) right-angle scattering, and (c) backscattering.

3(c) and 4(a), 4(b), and 4(c), where the temperature dependences of the Brillouin shift for the six longitudinal phonons propagating along [010] and [001] for small-angle scattering, right-angle scattering, and backscattering are illustrated with an expanded temperature scale together with the phonon decay rates $\Gamma / \pi$. The temperature dependences of the Brillouin shift and the decay rate for the right-angle scattering and backscattering along [010] are quite similar. In small-angle scattering for phonons along [010], the temperature dependence of the Brillouin shift is also similar to the others but the decay rate is not. Because relative error for the small-angle scattering is larger than those for the right-angle scattering and backscattering, the measured decay rate for the small-angle scattering is not very meaningful. Our measurement is limited not only by the resolution of the Brillouin spectrometer but also by the broadening of the incident-laserlight source and the broadening of the collection angle. The temperature dependences of the Brillouin shift and the decay rate for small-angle, right-angle scattering, and

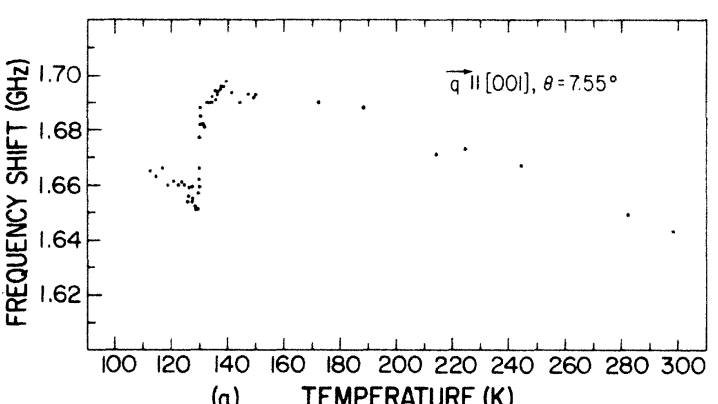

(a) TEMPERATURE (K)

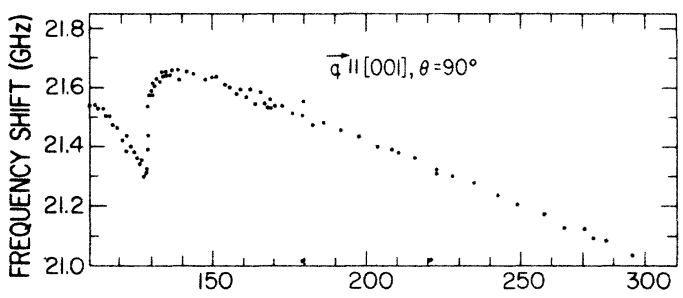

(b) TEMPERATURE (K)

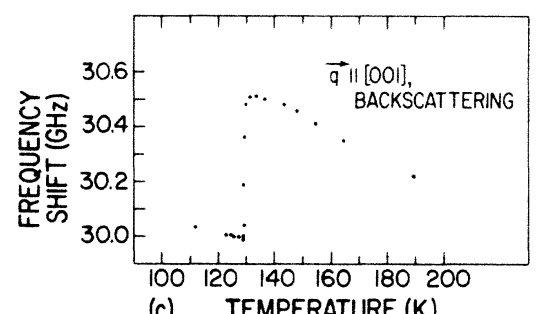

FIG. 2. Temperature dependences of Brillouin shifts of longitudinal phonons propagating along the $\mathrm{c}$ axis for (a) small-angle scattering, (b) right-angle scattering, and (c) backscattering.

backscattering for phonons along [001] show analogous behavior. There is a very narrow peak in the temperature dependence of the phonon decay rate around the phasetransition temperature $T_{c}$. That means that the phonons along [001] decay very quickly near $T_{c}$, unlike those along [010].

\section{B. Analysis of frequency shifts}

The TSCC crystal structure is orthorhombic, so the velocities of the phonons propagating along [010] and [001] are $v_{22}=\left(C_{22} / \rho\right)^{1 / 2}$ and $v_{33}=\left(C_{33} / \rho\right)^{1 / 2}$, respectively. Here, $C_{22}$ and $C_{33}$ are elastic-stiffness-tensor elements and $\rho=1.533 \mathrm{~g} / \mathrm{cm}^{3}$. The Brillouin shift is given by

$$
\Delta v=(v v / c)\left(n_{i}^{2}+n_{s}^{2}-2 n_{i} n_{s} \cos \theta\right)^{1 / 2},
$$

where $v$ is the frequency of the incident light, $v$ is the sound velocity, $c$ is the velocity of light in vacuum, $n_{i}$ and $n_{s}$ are the refractive indices for the incident and scattered light, respectively, and $\theta$ is the scattering angle.

For small-angle scattering, right-angle scattering, and backscattering for the [010] phonons, the approximate geometries are $x(z z) x, x+y(z z) x-y$, and $y(z z)-y$, respectively. The refractive index ellipsoid for TSCC is i1lustrated in Fig. 5. The polarization directions of the in- 

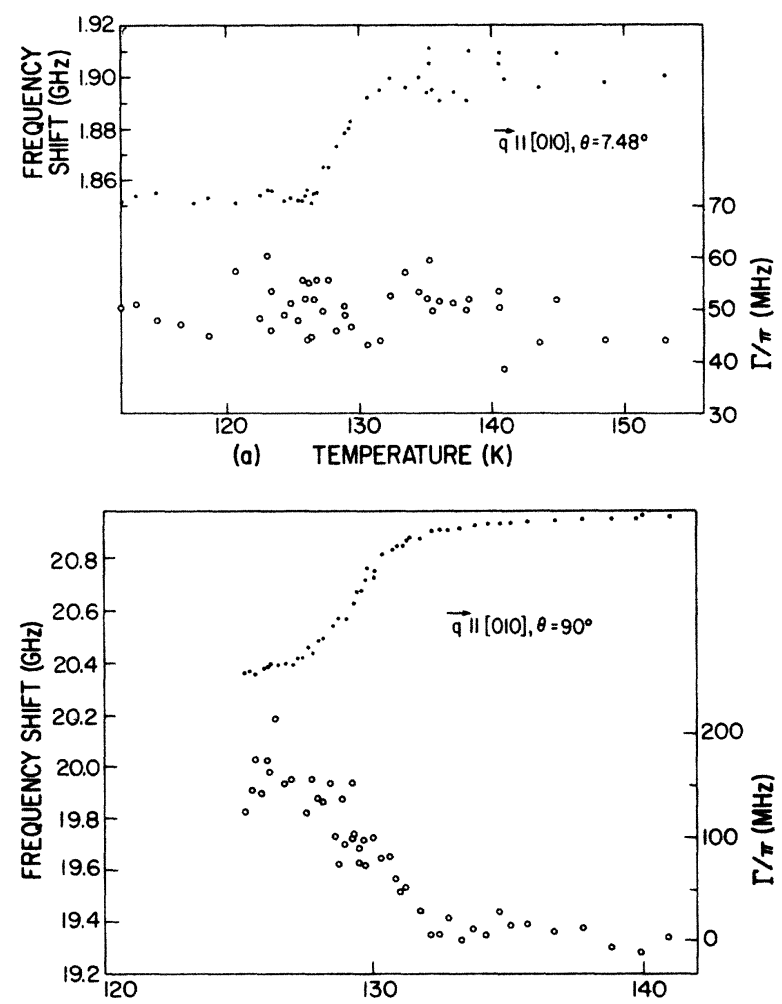

(b) TEMPERATURE (K)

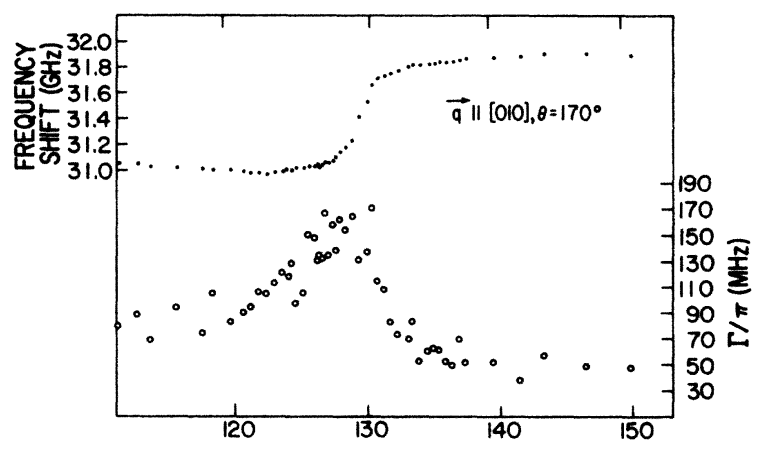

(c) TEMPERATURE (K)

FIG. 3. Temperature dependences near $T_{c}$ of Brillouin shift (C) and linewidth (O) (FWHM) of longitudinal phonons propagating along [010] for (a) small-angle scattering, (b) right-angle scattering, and (c) backscattering.

cident and scattered light in all three cases are along c. Thus $n_{i}=n_{s}=n_{c}=1.553$ (Ref. 15) above, and the frequency shift becomes

$$
\Delta v=2\left(v v n_{c} / c\right) \sin (\theta / 2) \text {. }
$$

For the right-angle scattering the Brillouin shift is given by

$$
\Delta v=\sqrt{2}\left(v n_{c} / c\right) \sin (\theta / 2) .
$$

The measured variation ${ }^{15}$ in $n$ between room temperature and $-150^{\circ} \mathrm{C}$ is about $0.8 \times 10^{-3}$, which is small and can be ignored. The sound velocity for small-angle scattering, right-angle scattering, and backscattering along both the $b$
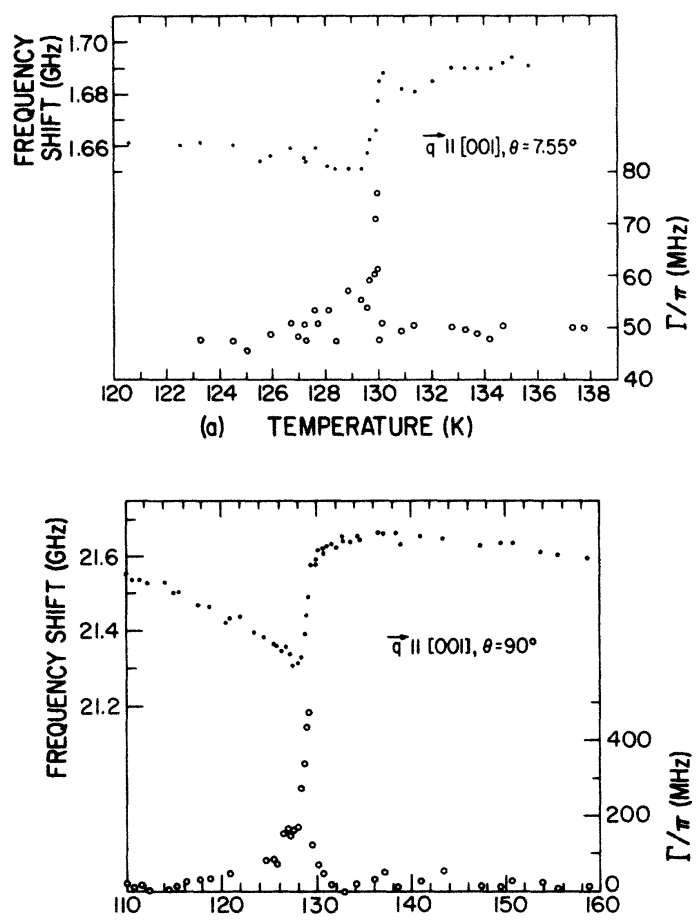

(b) TEMPERATURE (K)

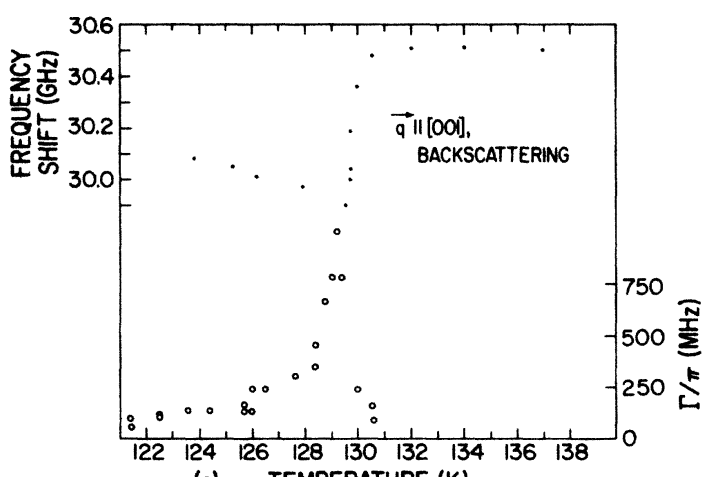

(c) TEMPERATURE (K)

FIG. 4. Temperature dependences near $T_{c}$ of Brillouin shift (O) and linewidth (O) (FWHM) of longitudinal phonons propagating along [001] for (a) small-angle scattering, (b) right-angle scattering, and (c) backscattering.

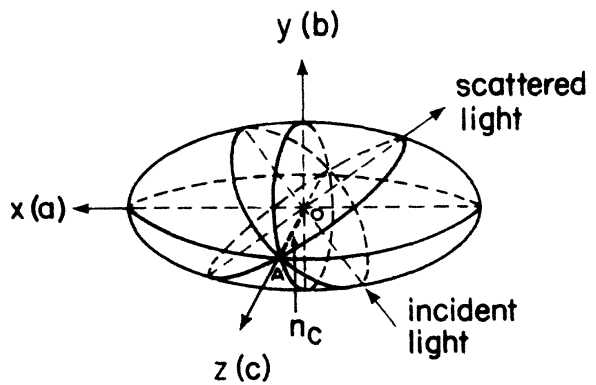

FIG. 5. The refractive index ellipsoid for TSCC, showing scattering geometry for right-angle scattering to observe [010] phonons. 
and c axes is calculated from Eq. (2) and the results are shown in Figs. 6 and 7. However, the scattering geometries for [001] phonons are approximately $x(y y) x$ for small-angle scattering and $y+z(x x) y-z$ for rightangle scattering, so $n_{c}$ in Eqs. (2) and (2') must be replaced by $n_{b}=1.551$ and $n_{a}=1.592$, respectively.

The three plots in Fig. 6 have very similar shapes. The phase transition is marked by about the same magnitude of rounded drop in velocity with decreasing temperature in all three cases, but, surprisingly, the sound velocities in these three cases at a given temperature are different. The same remarks apply to Fig. 7. There is the important difference that in Fig. 6 the backscattering velocity is higher than the other two, while in Fig. 7 the smallangle-scattering velocity is lower than the other two. We consider three possible origins for this apparent variation of the sound velocities for the different Brillouin scattering angles.

First, there is the error in determination of the scattering angle inside the crystal. In our experiments this error is at most $\pm 0.5^{\circ}$. From Eq. (2) the corresponding fractional error in velocity is

$$
\Delta v / v=(d v / d \theta) \Delta \theta / v=-0.00436 \cot (\theta / 2) .
$$

For our scattering angles of $7.48^{\circ}, 90^{\circ}$, and $170^{\circ}$, these velocity errors are $6.68 \%, 0.44 \%$, and $0.04 \%$, respectively. It is barely possible to account for the $14 \%$ lower velocity measured for the [001] phonons by small-angle scattering as shown in Fig. 7, if we assume the angular error is $1^{\circ}$ instead of $0.5^{\circ}$, but it is impossible to account in this way for the $8 \%$ difference in [010] phonon velocities measured by right-angle scattering and by backscattering.

A second possibility is that we assumed the wrong order in interpreting our Brillouin scattering spectra. Such a blunder is almost impossible for the small-angle scattering spectra. For the backscattering case we checked to insure that the correct order was assumed. Also, the backscattering results of Smolensky et al. for [010] phonon velocities agree quite well with ours, if we use their published ${ }^{14}$ elastic constant curve versus temperature and the

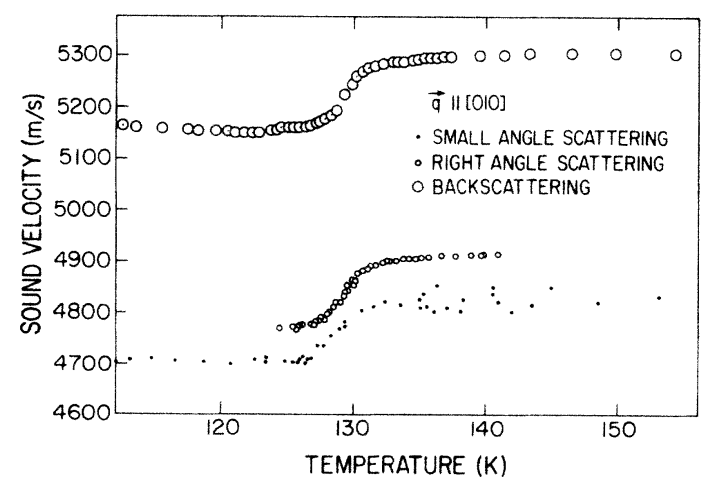

FIG. 6. Temperature dependences of sound velocity along [010] for small-angle scattering, right-angle scattering, and backscattering. The right-angle-scattering data shown here and in Figs. 1 and 3 have been presented previously (Ref. 2).

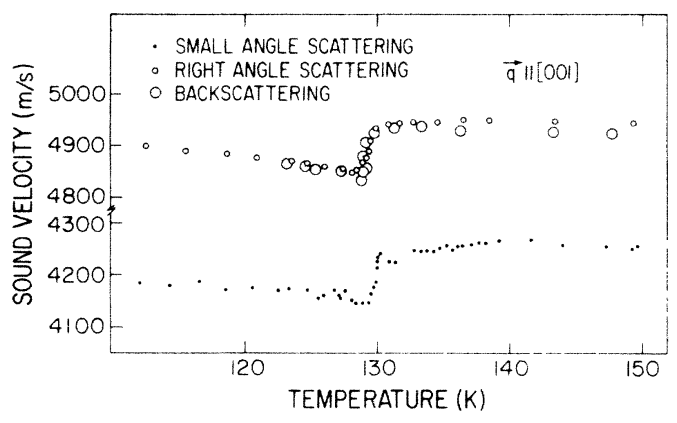

FIG. 7. Temperature dependences of sound velocity along [001] for small-angle scattering, right-angle scattering, and backscattering. The data for right-angle scattering (Ref. 12) and backscattering (Ref. 14) shown here and in Figs. 2 and 4 have been presented previously.

density $1.553 \mathrm{~g} / \mathrm{cm}^{3}$ for TSCC. In this way, we calculate $5230 \mathrm{~m} / \mathrm{s}$ for their highest sound velocity above $T_{c}$ (also found using an argon-ion laser), while we found 5270 $\mathrm{m} / \mathrm{s}$. However, in another paper, ${ }^{16}$ apparently based on the same experimental data, they report maximum sound velocity of $5090 \mathrm{~m} / \mathrm{s}$ for this phonon.

The only remaining possibility is that this dispersion of sound velocity actually occurs. This means that there is some coupling to another mode that is not affected significantly by the ferroelectric phase transition. We do not know the nature of this mode. It could be a dissipative mode associated with an incipient transition to another phase. There are two other phases, one known and the other proposed, for TSCC. The other known phase occurs only at elevated hydrostatic pressure and is presumably antiferroelectric. ${ }^{17}$ The hypothesis of a hexagonal parent phase is based on the observation that uniaxial pressure at elevated temperature can reorient the ferroelastic domains in the pseudohexagonal paraelectric phase by $60^{\circ}$ steps. ${ }^{3}$ However, as temperature is raised the crystal decomposes without reaching such a phase transition.

\section{Electrostrictive coupling}

For a longitudinal phonon propagating along [010], polarization fluctuations do not couple bilinearly to the strain, because of the appearance of the depolarization field in the longitudinal polarization wave. ${ }^{12}$ Therefore, the anomalies in the velocity and attenuation of these phonons should be explained by quadratic coupling of the polarization to the strain (electrostriction) in both the PE and FE phases.

For this analysis, we use the sound velocities obtained from Brillouin backscattering and from ultrasonic measurements ${ }^{11}$ for $v_{\infty}$ and $v_{0}$, respectively. We choose $T-T_{c}=0.9 \mathrm{~K}$ for the PE region and $T_{c}-T=1.3 \mathrm{~K}$ for the $\mathrm{FE}$ region. Accordingly, $v_{\infty}=5272 \mathrm{~m} / \mathrm{s}$ for the $\mathrm{PE}$ region and $5193 \mathrm{~m} / \mathrm{s}$ for the $\mathrm{FE}$ region, while $v_{0}=4780$ $\mathrm{m} / \mathrm{s}$ for the $\mathrm{PE}$ region and $4739 \mathrm{~m} / \mathrm{s}$ for the $\mathrm{FE}$ region.

The relation between the phonon decay rate $\Gamma$ or the Brillouin linewidth (FWHM) $\Gamma / \pi$ and the attenuation coefficient $\alpha$ is given by ${ }^{12}$

$$
\Gamma / \pi=\alpha v / \pi \text {. }
$$


Then,

$$
\alpha=(\Gamma / \pi) /(v / \pi) \text {. }
$$

From Fig. 3(c), at the temperatures $T-T_{c}=0.9$ and 1.3 $\mathrm{K}$, we have $\Gamma / \pi=130 \mathrm{MHz}, v=5272 \mathrm{~m} / \mathrm{s}$ and $\Gamma / \pi=100$ $\mathrm{MHz}, v=5193 \mathrm{~m} / \mathrm{s}$, respectively. Substituting these values into Eq. (4), we obtain

$\alpha=774.6 \mathrm{~cm}^{-1}$ at $T-T_{c}=0.9 \mathrm{~K}$ (in the PE region), $\alpha=967.9 \mathrm{~cm}^{-1}$ at $T_{c}-T=1.3 \mathrm{~K}$ (in the FE region) .

The relaxation between the attenuation $\alpha$ and the velocity $v$ is given by

$$
\alpha=\left[\left(v_{\infty}^{2}-v_{0}^{2}\right) / 2 v^{3}\right]\left[\omega^{2} \tau_{0} t^{-1} /\left(1+\omega^{2} \tau_{0}^{2} t^{-2}\right)\right],
$$

where $t=\left|\left(T-T_{c}\right) / T_{c}\right|$.

Substituting the attenuation-coefficient values from ultrasonic measurements at the temperatures $T-T_{c}$ $=+0.9$ and $-1.3 \mathrm{~K}(2.7$ and $11.1 \mathrm{~dB} / \mathrm{cm}$, respectively), the frequency $20 \mathrm{MHz}$, and the above values for $v_{\infty}$ and $v_{0}$, we obtain from Eq. (5) the values

$$
\begin{aligned}
& \tau_{0}^{E}=5.25 \times 10^{-13} \mathrm{~s} \text { for } T>T_{c}, \\
& \tau_{0}^{E}=3.32 \times 10^{-12} \mathrm{~s} \text { for } T<T_{c} .
\end{aligned}
$$

Here we designate the relaxation time as $\tau_{0}^{E}$, where the $E$ signifies that this relaxation time is related to energy rather than polarization fluctuations. We use the values of $\tau_{0}$ obtained above to estimate the attenuation coefficients for the longitudinal phonon along [010] for backscattering. We obtain

$\alpha=2209 \mathrm{~cm}^{-1}$ at $T-T_{c}=0.9 \mathrm{~K}$ (in the PE region), $\alpha=456 \mathrm{~cm}^{-1}$ at $T_{c}-T=1.3 \mathrm{~K}$ (in the PE region).

The calculated values of $\alpha$ agree well with those observed $\left(774.6 \mathrm{~cm}^{-1}\right.$ at $T-T_{c}=0.9 \mathrm{~K}$ and $967.9 \mathrm{~cm}^{-1}$ at $T_{c}-T=1.3 \mathrm{~K}$. Although the estimated attenuation coefficient $\alpha$ for $T-T_{c}=0.9 \mathrm{~K}$ is larger than that observed by us in backscattering, the estimated $\alpha$ for $T_{c}-T=1.3$ $\mathrm{K}$ is smaller than observed. These results differ from those of Hikita et al. ${ }^{12}$ for right-angle scattering.

\section{Bilinear polarization-strain coupling induced by spontaneous polarization}

If the polarization and strain couple bilinearly (Landau-Khalatnikov type) in the FE phase for the longitudinal phonons propagating along [100] and [001], the velocity $v$ in that phase is given by ${ }^{10}$

$$
v^{2}=v_{\infty}^{2}-\left(v_{\infty}^{2}-v_{0}^{2}\right) /\left(1+\omega^{2} \tau_{0}^{2} t^{-2}\right) .
$$

From Eq. (6) we expect that the transition is marked by a step down in velocity (or frequency shift) with decreasing temperature. Experimentally, the [001] phonons do not exhibit a sharp step at $T_{c}$, but show a slow rounding instead. These anomalies are attributed to the quadratic coupling of polarization with strain; in other words, electrostrictive coupling in the PE phase. ${ }^{12}$ In the FE phase, the fact that the maximum of the attenuation coefficient does not occur at the transition temperature $T_{c}$ but rather somewhat below $T_{c}$ indicates that the anomalous behaviors of the sound velocity and attenuation are caused by the piezoelectric coupling of the polarization with phonons ${ }^{18,19}$ propagating along [001]. In this case the relation between the velocity and the attenuation is given by Eq. (5). From Eqs. (5) and (6) we obtain the relation

$$
\left(T_{c}-T_{m}\right) / T_{c}=\omega \tau_{0},
$$

where $T_{m}$ is the temperature at which the decay rate of the phonon is maximum, and $T_{c}=130 \mathrm{~K}$.

For [001] phonons observed by small-angle scattering, we found $T_{c}-T_{m}=0.072 \mathrm{~K}$. Substituting this value into Eq. (7), we obtain

$$
\tau_{0}=5.25 \times 10^{-14} \mathrm{~s} .
$$

This agrees quite well with the result $\left(2.7 \times 10^{-13} \mathrm{~s}\right)$ obtained by Hikita et al. ${ }^{12}$ for right-angle scattering.

\section{E. Concluding remarks}

As usual, the most interesting features of Brillouin scattering in TSCC are the anomalies in velocity and decay rate of [010] longitudinal phonons which we obtained by small-angle scattering, right-angle scattering, and backscattering. Unfortunately, the decay rate for small-angle scattering is too small to measure very accurately. Nevertheless, this decay rate seems to be not very different from those obtained from backscattering anc right-angle scattering. The dip in velocity just below $T_{c}$ and the rounding just above $T_{c}$ are features for which a quantitative theory is needed. Also needed are additional measurements at other laser frequencies to obtain more points on the phonon dispersion curves.

Finally, TSCC is claimed to exhibit tricritical behavior at high pressure. ${ }^{20}$ Examination of the anomalies in the velocity and the critical index of the relaxation time around this tricritical point would be very interesting.

\section{ACKNOWLEDGMENTS}

We thank Dr. Tomoyuki Hikita for his helpful suggestions, Professor Robert Gammon for a valuable discussion, and Paul Schnackenberg for his experimental assistance. This work was supported in part by National Science Foundation Grant No. DMR-82-05280.
${ }^{1}$ (a) R. Pepinsky and Y. Makita, Bull. Am. Phys. Soc. 7, 241 (1962); (b) T. Ashida, S. Bando, and M. Kakudo, Acta Crystallogr. Sect. B 28, 1560 (1972).

${ }^{2}$ T. Hikita, J. T. Wang, P. T. Schnackenberg, and V. H. Schmidt, Jpn. J. Appl. Phys. 24, Suppl. 24-2, 494 (1985).

${ }^{3}$ A. Sawada, Y. Makita, and Y. Takagi, J. Phys. Soc. Jpn. 42,
1918 (1977)

${ }^{4}$ T. Chen and G. Schaack, J. Phys. C 17, 3801 (1984); 17, 3821 (1984).

${ }^{5}$ Y. Makita, J. Phys. Soc. Jpn. 20, 2073 (1965).

${ }^{6}$ E. Sandvold and E. Courtens, Phys. Rev. B 27, 5660 (1983).

${ }^{7}$ M. A. Pérez Jabindo, A. Lopez Echarri, and M. J. Tello, Fer- 
roelectrics 39, 1171 (1981).

8J. Lajzerowicz and J. F. Legrand, Phys. Rev. B 17, 1438 (1978).

${ }^{9}$ K. V. Kozlov, A. A. Volkov, J. F. Scott, G. E. Feldkamp, and J. Petzelt, Phys. Rev. B 28, 255 (1983).

${ }^{10} \mathrm{G}$. E. Feldkamp, J. F. Scott, and W. Windsch, Ferroelectrics 39, 1163 (1981).

${ }^{11} \mathrm{G}$. Sorge and U. Straube, Ferroelectrics 21, 533 (1978).

${ }^{12}$ T. Hikita, P. Schnackenberg, and V. H. Schmidt, Phys. Rev. B 31, 299 (1985).

${ }^{13}$ M. Yamada and V. H. Schmidt, Rev. Sci. Instrum. 49, 1226 (1978).

14G. A. Smolensky, I. G. Siny, A. K. Tagantsev, S. D. Prokhorova, and W. Windsch, Ferroelectrics 64, 221 (1985).
${ }^{15}$ N. R. Ivanov and H. Arndt, Kristallografiya 24, 508 (1979) [Sov. Phys.-Crystallogr. 24, 291 (1979)].

${ }^{16}$ G. A. Smolensky, I. G. Siny, A. K. Tagantsev, S. D. Prokhorova, V. D. Mikvabiya, and W. Windsch, Pis'ma Zh. Eksp. Teor. Fiz. 39, 307 (1984) [JETP Lett. 39, 368 (1984)].

${ }^{17}$ V. H. Schmidt, Solid State Commun. 35, 649 (1980).

${ }^{18}$ R. W. Gammon and H. Z. Cummins, Phys. Rev. Lett. 17, 193 (1966).

${ }^{19}$ T. Yagi, M. Tokunaga, and I. Tatsuzaki, J. Phys. Soc. Jpn. 40, 1659 (1976).

${ }^{20}$ H. Reichelt, W. Windsch, and A. Sienkiewicz, Ferroelectrics 34, 195 (1981). 